\title{
Outcome of Skin Graft in Postburn Finger Contractures: An Integrated Technique of Evaluation
}

\author{
A ALI $^{\mathrm{a}}$, SH KHUNDKAR ${ }^{\mathrm{b}}$
}

\begin{abstract}
Summary:
Objective: This prospective clinical study was conducted between July 2003- June 2005 at Dhaka Medical College Hospital, Dhaka, Bangladesh to make a comparative evaluation of the outcome of skin graft on patients of postburn contractures of the fingers using integrated scores for measuring several outcomes together.

Materials \& Methods: A total of 56 subjects of postburn contractures of the fingers were selected consecutively and were evaluated at baseline by number of digits affected, surface area involved, extension deficit, fingers with maximum extension deficit and duration of contracture. Thirty two subjects were assigned to Full-thickness skin graft group (Group-A) and 24 to Split-thickness skin graft group (Group-B). Respective graft coverage was applied to the wound following release of contracture. Immediate outcome was evaluated in terms of graft take and number of graft-site complications, while follow up outcome was evaluated in terms of extension deficit six months after correction using specific scores for defined outcome.
\end{abstract}

Results: Over $70 \%$ of the subjects were $<15$ years with mean ages of Group-A and Group-B were $9.38 \pm 1.66$ and $9.94 \pm 1.42$ years respectively. In both groups maximum extension deficit was found in little finger (78\% in Group-

Introduction:

Hand is a highly specialized organ, as it has grasping pinching and hooking functions, carried out by musculotendinous unit. It can give information about position, size and shape of an object by its highly developed sensory mechanism and described as third

a. Dr. Ali Ayub, FCPS, MS, Asst. Professor, Dept. of Plastic Surgery, Chittagong Medical College \& Hospital (CMC \& H), Bangladesh.

b. Prof. Shafquat H Khundkar, FCPS; FICS (Plastic), Professor \& Head, Plastic Surgery, Dhaka Medical College \& Hospital, Bangladesh

Address of Correspondence: Dr. Md. Ayub Ali, Asst. Professor, Dept. of Plastic Surgery, Chittagong Medical College \& Hospital, Chittagong, Bangladesh. E-mail: ayub59@gmail.com; Mobile: 0088- 01819-226373.

Received: 24 February, 2008

Accepted: 29 September, 2008
$A$ and 54\% in Group-B). The Interphalangeal (IP) joints were observed to be most frequently involved $(78 \%$ in Group-A and 75\% in Group-B). The duration of contracture, number of digits involved and surface area of the fingers involved were almost identically distributed between the groups. Outcome shows that donor-site morbidities (discolouration and hypertrophic scars) were significantly less in Group-A compared to that in Group$B(p<0.001$ and $p=0.047)$. Similarly the Group-A was significantly superior compared to Group-B in terms of minimal extension deficit $\left(0-10^{\circ}\right) 6$ months after correction (59.4\% vs. $25 \%, P<0.05)$. About two-third of the subjects in both the groups demonstrated 100\% graft take and around $80 \%$ had 2 or $<2$ graft-site morbidities. The excellent outcome was significantly higher in GroupA (37.5\%) than that in Group-B (12.5\%) $(p<0.05)$.

Conclusion: Full-thickness skin graft is a better option of intervention than Split-thickness skin graft for coverage after release of contracture. However, the findings need to he validated by a larger sample size.

Key words: Postburn flexion deformity, full-thickness skin graft, split-thickness skin graft, graft take, donor-site morbidity, graft-site morbidity and extension deficit.

(J Bangladesh Coll Phys Surg 2009; 27: 25-29)

eye $\mathrm{e}^{1}$. Hand function is grossly impaired if post burn deformity occurs, specially involving fingers and palm. For postburn deformity of hand, the goal of treatment is recovery of functionally acceptable digital motion with intact sensation ${ }^{2}$. There are several options of resurfacing after release of postburn contracture of fingers. Incisional release and grafting is usually preferable to excision and grafting, particularly if dorsal scarring is fresh and hyperemic ${ }^{3}$. As vast majority of burns result in loss of skin, replacement with skin graft rather than flap is the most appropriate method of coverage ${ }^{4}$. Full-thickness skin graft is usually recommended in preference to split-thickness skin graft for resurfacing after release of postburn contractures ${ }^{5}$. The present study was intended to make a comparative evaluation of the 
outcome of skin graft on patients of postburn contractures of the fingers using integrated scores for measuring several outcomes together.

\section{Materials \& methods:}

The study was conducted during the period from July 2003 - June 2005 at Plastic Surgery Department of Dhaka Medical College Hospital. A total of 56 patients were selected consecutively from the patients seeking treatment for postburn flexion deformity of fingers. The patients were evaluated at baseline by number of digits affected, surface area involved, extension deficit, fingers with maximum extension deficit and duration of contracture. Following random allocation procedure 32 patients were assigned to Full-thickness skin graft group (Group-A) and 24 to Split-thickness skin graft group (Group-B). Patients of postburn flexion deformity of fingers irrespective of age and sex, with scarring on the flexor surface of fingers and Volar contractures were included in the study. The criteria that refrained patients from participating were contracture over $100^{\circ}$, extensive scar demanding flap coverage and stiffness of joints.

With all aseptic precautions and after applying tourniquet, incision was made over the most contractured site and proceeded to the sides making it a 'fish-tail appearance'. In case of contracture bands a small 'Z' plasty was added. Release of contracture was done at the satisfactory level with preservation of digital vessels and nerves. Full-thickness skin graft was harvested from groin or upper and medial aspect of arm, while split-thickness skin graft was harvested from thigh. Respective graft coverage was applied to the wound. In case of full-thickness skin graft, donor site was closed intradermally and in case of split-thickness skin harvesting, only sterile dressings were applied on the donor site. In children $<12$ years, immobilization was done by needle ( 22 gauze) in extensor subtendinous space. In patients 12 or $>12$ years old, fingers were immobilized by Kirschner wire introduced through pulp and passing across the interphalangeal joint before placement of the graft on the recipient site.

Immediate outcome was evaluated in terms of graft take and number of graft-site complications. Follow up evaluation was done six months after correction. Outcome during follow up was evaluated in terms of extension deficit measured using a Goniometer. As there were several outcomes, it was difficult to decide which group had better outcome unless all the outcomes were weighted together. Therefore, to compare the outcomes between the two groups, all the individual outcomes were scored based on their merits and added together to find an integrated score of 9 (Table I). The score 3 or below was considered poor and from $9-8$ was considered excellent outcomes, while fair $(5-4)$ and good $(7-6)$ were in between them. The test statistics used to analyze the data were descriptive statistics Student's t-test, Chisquared Test and Fisher's Exact Probability Test.

\section{Results:}

The findings of the study showed that age, sex and other baseline variables were almost identically distributed in both the groups (Table II). More than $70 \%$ of the subjects were below 15 years of age with mean ages of Group-A and Group-B were $9.38 \pm 1.66$ vs. $9.94 \pm 1.42$ years respectively. Over three-quarter $(78.1 \%)$ of the subjects in Group-A and two-third $(66.7 \%)$ in Group-B had extension deficit from 45 $90^{\circ}$ with maximum extension deficit being in little fingers (78\% in Group-A and 54\% in Group-B) (Fig.1). The IP joints were found most frequently involved ( $78 \%$ in Group-A and $75 \%$ in Group-B). Both the groups were found almost identical with respect to duration of contracture (11.0 \pm 6.24 vs. 9.0 \pm 3.05 months, $p>0.05)$ number of digits involved $(2.0 \pm 0.17$ vs. $2.0 \pm 0.18, \mathrm{P}>0.05)$ and flexor surface affected $(21.88 \pm 9.98 \%$ vs. $22.92 \pm 9.08 \%, \mathrm{p}>0.05)$. About two-third of the patients in both the groups demonstrated $100 \%$ graft take. Around $80 \%$ of the patients in both the groups had 2 or $<2$ graft-site morbidities. However, donor-site morbidities (in terms of discoloration and hypertrophic scars) were found to be significantly less in Group-A compared to Group-B ( $<0.001$ and $p=0.047)$. Similarly the Group-A was observed to be significantly superior compared to Group-B in terms of minimal extension deficit $\left(0-10^{0}\right)$ six months after correction $(59.4 \% v s .25 \%, \mathrm{p}<0.05)$ (Table III). The frequency of donor-site infection and recurrence of contracture were not significantly different between the groups $(\mathrm{p}$ $>0.05$ ). The integrated score showed that excellent outcome was significantly higher in Group-A $(37.5 \%)$ relative to Group-B $(12.5 \%) \quad(p<0.05)$ (Table IV). The good, fair and poor outcomes in Group-A were $50 \%, 9.4 \%$ and $3.1 \%$ respectively, while in Group-B the same were $54.2 \%, 20.8 \%$ and $12.5 \%$ respectively (Fig.-2). 


\section{Table-I}

\section{Scoring system for outcome evaluation}

Outcome variables

Graft take

Number of graft site complications

Extension deficit six months

after correction

\begin{tabular}{ccccc}
\multicolumn{5}{c}{ Scores assigned } \\
\hline 4 & 3 & 2 & 1 & 0 \\
- & - & $100 \%$ & $<100 \%$ & - \\
- & $0-2$ & $3-5$ & $>5$ & -
\end{tabular}

$\begin{array}{lllll}0-10^{\circ} & 11^{\circ}-20^{\circ} & 21^{\circ}-30^{\circ} & 31-40^{\circ} & >40^{\circ}\end{array}$

Table-II

\section{Distribution of baseline variables between groups}

Baseline variables

Age (years)\#

Sex 9

Male

Female

Duration of contracture (months)\#

Proportion flexor surface involved (\%)\#

No. of digits involved\#

Joints involved

IP joints only

Both IP and MP joints

Extension deficit (degree)

$45-90$

$>90$

\begin{tabular}{cc}
\multicolumn{2}{c}{ Group } \\
\hline Group A $(\mathrm{n}=32)$ & Group B $(\mathrm{n}=24)$ \\
$2.0 \pm 0.17$ & $2.0 \pm 0.18$
\end{tabular}

p-values

0.339

$17(53.1)$

$15(62.5)$

0.339

15(46.9)

9(37.5)

$11.0 \pm 6.24$

$9.0 \pm 3.05$

0.56

$21.88 \pm 9.98$

$22.92 \pm 9.08$

0.603

$2.0 \pm 0.17$

$2.0 \pm 0.18$

0.339

22(68.8)

18(75.0)

0.608

10(31.3)

6(25.0)

25(78.1)

$17(70.9)$

7(29.2)

\# Data were analysed using Mann-Whitney Test and were presented as mean (6 SEM.

I Data were analysed using Chi-squared ( $(2)$ Test and presented as frequency with corresponding (\%).

\section{Table-III}

Comparison of outcomes between groups:

Outcomes

Graft take (100\%)

No. of graft-site morbidities ( $£ 2$ )

Extension deficit 6 months after correction

$0-100$

$11-200$

$21-300$

\begin{tabular}{ccc}
\multicolumn{2}{c}{ Group } & p-values\# \\
\cline { 1 - 2 } Group-A (n=32) & Group-B (n=24) & \\
$21(65.6)$ & $16(66.7)$ & 0.935 \\
$26(81.3)$ & $19(79.1)$ & 0.747 \\
$19(59.4)$ & $6(25.0)$ & $0.034 \mathrm{~S}$ \\
$10(31.3)$ & $15(62.5)$ & \\
$3(9.4)$ & $3(12.5)$ &
\end{tabular}

\# Data were analysed using $\mathrm{c}^{2}$ Test or Fisher's Exact Test and presented as n(\%); $\mathrm{S}=$ Significant 


\section{Table-IV}

Comparison of total outcome scores between groups:

\begin{tabular}{lcccc} 
Outcome variables & Highest scores & \multicolumn{2}{c}{ Scores attained by } & p-values\# \\
\cline { 3 - 4 } & & Group-A $(\mathrm{n}=30)$ & Group-B $(\mathrm{n}=25)$ & \\
Graft take $(100 \%)$ & 2 & $1.66 \pm 0.48$ & $1.67 \pm 0.48$ & 0.937 \\
Graft site morbidity & 3 & $2.81 \pm 0.40$ & $2.79 \pm 0.41$ & 0.849 \\
Extension deficit six months after correction & 4 & $3.53 \pm 0.62$ & $2.96 \pm 0.75$ & 0.003 \\
\hline Total outcome & 9 & $8.00 \pm 1.50$ & $7.42 \pm 2.50$ & $<0.001$ \\
\hline
\end{tabular}

\# Data were analysed using Student's t-test and presented as mean \pm SD.

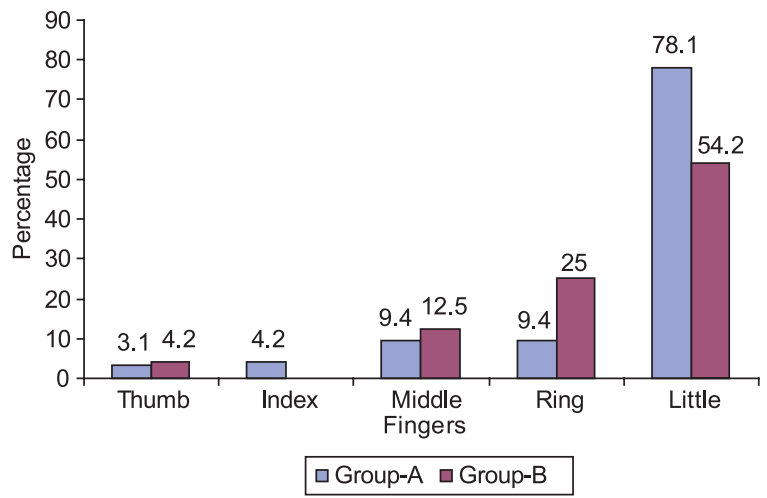

Percentage will not correspond to $100 \%$ because of multiple response

Fig. 1: Fingers with maximum extension deficit

\section{Discussion:}

Postburn flexion deformity of fingers is a common sequele of burn which demands restoration of manual function. The goal of surgical treatment as stated by Stricland et $a .^{2}$ should be the recovery of functionally acceptable digital motion with intact sensation.

In the present study, majority of the burn contractures of fingers was observed below the age of 15 years, $(59.4 \%$ in full-thickness and $79.3 \%$ in splitthickness). The youngest one was 1 year and the oldest one was 38 years old. A male preponderance was observed in both the groups bearing consistency with the other studies ${ }^{6,7}$. Full-thickness skin grafts were found to achieve the best functional results, which are similar to the studies of other investigators 5,8 . About two-third of the patients in both the groups had $100 \%$ graft take which was almost consistent with findings of Alexander et al. ${ }^{8}$, where $91 \%$ of the patients had $100 \%$ graft take.

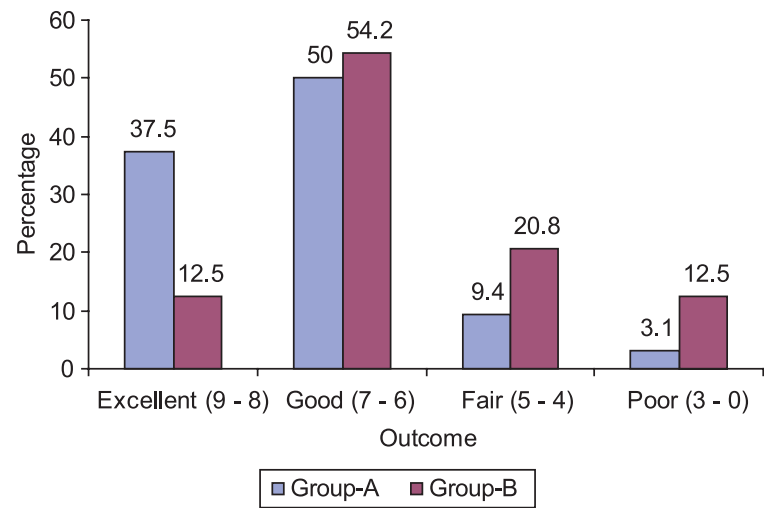

Fig. 2: Comparison of integrated outcome between groups

Donor-site morbidities were more frequently encountered by patients of split-thickness than those encountered by full-thickness; graft-site morbidities were almost equally distributed between groups (81.3 and $79.1 \%$ in full-thickness and split-thickness respectively, $p=0.747)$. Recurrence of contracture was considerably higher in split-thickness (45.8\%) compared to that in full-thickness $(21.9 \%)$ ( $\mathrm{p}=$ 0.057). Pigmentary changes were almost similar in both the groups $(78.1 \%$ and $75 \%$ in full-thickness and split-thickness respectively, $\mathrm{p}=0.784)$. The only disadvantage of split-thickness is its tendency to recur contracture, which was also found in the study of Iwuagwe et $a l^{5}$. Among donor site complications, about $96 \%$ patients of split-thickness noticed discolouration which was completely absent in subjects of full-thickness $(\mathrm{p}<0.001)$. This is because the donor-site of group-A was primarily closed. Hypertrophic scar was also significantly lower in fullthickness than that in split-thickness $(\mathrm{p}<0.05)$. 
Postoperative functional evaluation done six months after correction showed that about $60 \%$ of fullthickness had very minimum extension deficit $(0-$ $10^{0}$ ), In contrast, $62.5 \%$ of split-thickness showed moderate to severe extension deficit $\left(11^{0}-20^{0}\right)$, indicating superiority of full-thickness over splitthickness $(p=0.034)$. Similar result was also observed by the other investigators ${ }^{5}$, where they used full-thickness skin graft rather than split thickness skin graft. Alexander et al. ${ }^{8}$ and Burn \& $\mathrm{Oh}^{9}$ also demonstrated similar results. However, some authors held completely reverse opinion. Their results suggested that there is no long-term functional advantage in the use of full-thickness skin graft to resurface the palmer surface following release of the burn scar. They felt that the use of split-thickness skin graft which provide identical long-term functional result are less deforming and are superior cosmetically. ${ }^{10}$

\section{Conclusion:}

The hand is frequently involved as a part of much larger burn complex. Due to inadequate and improper initial care, a superficial burn is usually converted into a deep burn by gross secondary infection leading to slow healing and protracted illness with resultant contractures and scarring of the soft tissue. The purpose of surgical treatment is to obtain the best result with least complicated procedure in minimum time. As the vast majority of burns results in loss of skin only, skin replacement with skin graft is the method of choice for resurfacing. As split-thickness skin graft frequently contracts following resurfacing, full-thickness skin graft is the better option of coverage after release of postburn flexion contractures of the fingers. However, as the sample size was small in the present study, it requires to be validated by a larger sample size.

\section{Reference:}

1. Davies D. Plastic and Reconstructive surgery: the hand. $\mathrm{Br}$ Med J 1985;190:1650.

2. Strickland JW. Flexor tendon injuries. Orthop Rev 1986; $15: 632 / 2134 / 645$.

3. Rousso M, Wexler MR. Management of the burned hand. In: Goldwyn RM, editor. Long term results in plastic and Reconstructive surgery. Boston: Little, Brown and Company, 1980.

4. Beasley RW. Secondary repair of burned hands. Clin Plast Surg 1981;8:141 62.

5. Iwuagwu FC, Wilson D, Bailie F. The use of skin grafts in postburn contracture release: a 10 year review. Plast Reconstr Surg 1999;103:1198 204.

6. Iregbulem LM. Postburn volar digital contractures in Nigerians. Hand 1980; 12:54 61.

7. Stern PJ. Classification and management of burned thumb contractures in children. Burns 1985; 11:168 74.

8. Alexander JW, MacMillan BG, Martel L, Krummel R. Surgical correction of postburn flexion contractures of the fingers in children. Plast Reconstr Surg 1981;68:218 24.

9. Burm S, Oh SJ. Fist position for skin grafting on the dorsal hand. II. Clinical use in deep burns and burn scar contractures. Plast Reconstr Surg 2000;105:581 8.

10. Pensler JM, Steward R, Lewis SR, Herndon DN. Reconstruction of the burned palm: full thickness versus split thickness skin grafts long term follow up. Plast Reconstr Surg 1988;81:46 9. 